\title{
Quercetin protects against inflammation, MMP-2 activation and apoptosis induction in rat model of cardiopulmonary resuscitation through modulating Bmi-1 expression
}

\author{
DAWEI WANG $^{1 *}$, XIAOQIAN LOU ${ }^{2 *}$, XIAO-MING JIANG ${ }^{1}$, CHENXI YANG $^{3}$, \\ XIAO-LIANG LIU ${ }^{1}$ and NAN ZHANG ${ }^{1}$
}

\begin{abstract}
${ }^{1}$ Department of Emergency, The First Hospital of Jilin University; ${ }^{2}$ Department of Endocrinology, Second Department, The First Hospital of Jilin University, Changchun, Jilin 130021, P.R. China; ${ }^{3}$ Centre for Heart and Lung Innovation, University of British Columbia, Vancouver, BC V6P 2G9, Canada
\end{abstract}

Received January 13, 2016; Accepted January 24, 2018

DOI: $10.3892 / \mathrm{mmr} .2018 .8994$

\begin{abstract}
With extensive pharmacological actions, quercetin has anti-oxidant, free radical scavenging, anti-tumor, anti-inflammatory, anti-bacterial and anti-viral activity. Quercetin also reduces blood glucose and reduces high blood pressure, and has immunoregulation and cardiovascular protection functions. Additionally, it has been reported that it can reduce depression. The current study evaluated whether quercetin protects against inflammation, matrix metalloproteinase-2 (MMP-2) activation and apoptosis induction in a rat model of cardiopulmonary resuscitation (CPR), and whether Bmi-1 expression was involved in the effects. In CPR model rats, treatment with quercetin significantly recovered left ventricular ejection fraction, left ventricular fractional shortening, ejection fraction (\%), and left ventricle weight/body weight. Treatment with quercetin significantly inhibited ROS generation, inflammation and MMP-2 protein expression in the rat model CPR. Finally, quercetin significantly suppressed caspase- 3 activity and activated Bmi-1 protein expression in the rat model of CPR. The results demonstrated that quercetin protects against inflammation, MMP-2 activation and apoptosis induction in a rat model of CPR, and that this may be mediated by modulating Bmi-1 expression.
\end{abstract}

Correspondence to: Dr Xiao-Liang Liu or Mr. Nan Zhang, Department of Emergency, The First Hospital of Jilin University, 71 Xinmin Street, Chaoyang, Changchun, Jilin 130021, P.R. China E-mail: feilufangduan@126.com

E-mail: muchao57@163.com

${ }^{*}$ Contributed equally

Key words: quercetin, cardiopulmonary resuscitation, inflammation, matrix metalloproteinase-2, B-cell specific moloney leukemia virus insertion site 1

\section{Introduction}

Cardiopulmonary arrest is common seen in emergency practice. With the standardization of cardiopulmonary resuscitation (CPR), the rate of resuscitation is increased (1). However, $\sim 60 \%$ of patients experience cerebral resuscitation failure and $2-3 \%$ of patients that survive suffer from severe nerve function deficits (1). The prognosis of resuscitated patients depends on the levels of cerebral ischemic injuries (2). Reperfusion injury following complete cerebral ischemia is the major cause of cerebral injury. Following CPR, patients experience ischemia-reperfusion injury (3). It is established that the mechanisms of ischemia-reperfusion injury are associated with oxygen radicals and calcium overload (4). Following $\mathrm{CPR}$, various inflammatory cells are activated and produce cytokines that participate in the damaging effects of ischemia-reperfusion (5). At the early stage of CPR, the increase of tumor necrosis factor- $\alpha$ (TNF- $\alpha$ ) and interleukin-6 (IL-6) indicates that the inflammatory response system is activated following CPR. The release of cytokines can be considered as a stress response to cardiac arrest (6).

The uniform conduction of electrical activity depends on electrical coupling among myocardial cells and is influenced by the extracellular matrix (ECM) (7). Under normal physiological conditions, the synthesis and degradation of ECM is in dynamic equilibrium (8). Its excessive synthesis or abnormal degradation may alter the mechanical properties of myocardium and electrophysiological structures, which may affect its uniform conduction (9). Previous studies revealed that the regulation of matrix metalloproteinases (MMPs) have an important role in the synthesis and degradation of $\operatorname{ECM}(7,10)$.

B-cell specific moloney leukemia virus insertion site 1 (Bmi-1) is an important member of the Polycomb-group protein family (11). It has essential actions in repairing DNA damage, cell cycle control, the stability of chromatin, the activation of genetic transcription and apoptosis (12).

Patients with cardiac arrest have obvious central lesions following CPR. Following complete cerebral ischemia-reperfusion injury, hydromechanics, pathomorphology and metabolic 
disorders may form encephaledema, and severity influences patient prognosis (13). Clinic trials demonstrated that even when autonomic circulation recovered following CPR, some patients still experienced loss of consciousness (14).

Quercetin is a polyhydroxy flavonoid widely present in flowers, leaves and fruits, with various biological activities and high pharmaceutical value (15). Quercetin is present in $>100$ types of Chinese herbal medicine, including sophora flower bud, sophora flower, the root bark of the peony tree, chrysanthemum, Hypericum japonicum, Parasemia plantaginis, parasitic Loranthus, hairyvein agrimony, Perfoliate knotweed herb, Gynostemma pentaphyllum, maythorn, Hypericum perforatum, semen cuscutae, ginkgo leaf, Aesculus wilsonii rehd, Oldenlandia diffusa, cockscomb, Houttuynia cordata, emblic leafflower fruit, Saururus chinensis, Psidium guajava leaf, phyllan lhus mat shillirae, Chinese violet and Sedum sarmentosum (16-19). The results of the current study demonstrated that quercetin inflammation, MMP-2 activation and apoptosis induction in a rat model of CPR and investigated the mechanism of the protective effect of quercetin following CPR.

\section{Materials and methods}

Experimental animals. The protocol of animal experiments was approved by the University Laboratory Animal Research Committee of The First Hospital of Jilin University (Changchun, China). Adult Sprague-Dawley (SD) rats (250 $\pm 30 \mathrm{~g} ; \mathrm{n}=30$ ) were purchased from Qinghai Experimental Animal Center (Qinghai, China) and maintained in a specific pathogen-free environment. The CPR model was established by an asphyxia method and animals anaesthetized with intraperitoneal injection of chloral hydrate (Shanghai Ruiqi Biological Engineering Research Center, Shanghai, China). Tracheal intubation was closed at the end of expiration for 5 min. SD rats were randomly divided into sham operation group (sham; $n=6)$, quercetin group (QCT; $n=6)$, Model group (Model, $\mathrm{n}=8$ ) and Model +quercetin group (Model + QCT; n=8). In the QCT group and Model + quercetin group, normal or CPR model SD rats were treated with intragastric injection of $50 \mathrm{mg} / \mathrm{kg}$ quercetin once a day for 5 days, respectively. The chemical structure of quercetin is presented in Fig. 1 and was purchased from Sigma-Aldrich (Merck KGaA, Darmstadt, Germany). The rats of Model and QCT group were anaesthetized with $35 \mathrm{mg} / \mathrm{kg}$ pentobarbital sodium, tracheal intubation was closed at the end of expiration for $5 \mathrm{~min}$ and cardiopulmonary resuscitation was performed. The rats of sham group were anaesthetized with $35 \mathrm{mg} / \mathrm{kg}$ pentobarbital sodium without cardiopulmonary resuscitation.

Hemodynamic changes. After treatment with quercetin, surgical procedures were performed on all SD rats to measure hemodynamic parameters. Left ventricular dysfunction systolic (LVDs), left ventricular dysfunction diastolic (LVDd), stroke volume $(\mathrm{SV})$ and cardiac output $(\mathrm{CO})$. Ejection fraction $(\mathrm{EF} \%)$ and left ventricular shortening fraction (FS\%) was calculated using the same area-length method as previously described (19).

ELISA. Serum was separated from venous blood of each rat following intragastric injection treatment with quercetin and used to measure reactive oxygen species (ROS) generation

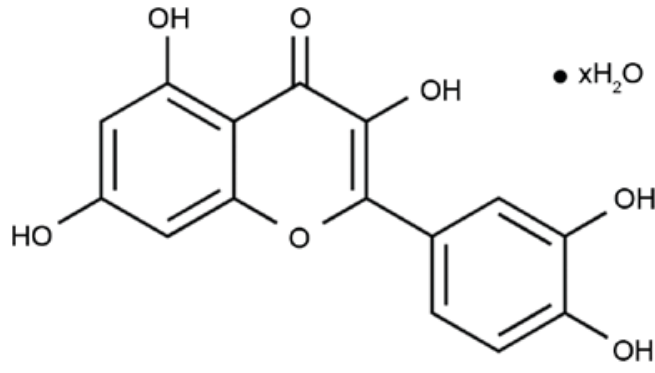

Figure 1. Chemical structure of quercetin.

(S0033), IL-6 (PI328) and TNF- $\alpha$ (PT516) activities using assay kits according to the manufacturer's instructions (Beyotime Institute of Biotechnology, Haimen, China). Caspase-3 activity was measured using a caspase-3 activity detection kit (C1116, Beyotime Institute of Biotechnology, Haimen, China).

Left ventricle weight/body weight. After 4 weeks, body weight was measured and recorded. Subsequently, left ventricle weight was acquired. Left ventricle weight/body weight was calculated.

Western blotting analysis. Heart tissue samples were homogenized in radioimmunoprecipitation assay buffer (10 $\mu \mathrm{g} / \mathrm{ml}$, Beyotime Institute of Biotechnology) in the presence of protease inhibitors (EMD Millipore, Billerica, MA, USA). The supernatant was collected to measure total proteins using the bicinchoninic acid method (Beyotime Institute of Biotechnology). Total proteins $(50 \mu \mathrm{g})$ were separated by $12 \%$ SDS-PAGE and transferred to nitrocellulose membranes. Following blocking with $5 \%$ non-fat milk in TBS-Tween at $37^{\circ} \mathrm{C}$ for $1 \mathrm{~h}$, membranes were incubated with anti-MMP-2 (sc-10736, 1:5,000; Santa Cruz Biotechnology, Inc., Dallas, TX, USA), anti-Bmi-1 (sc-10745, 1:2,000; Santa Cruz Biotechnology, Inc.), anti-inducible nitric oxide synthase (iNOS;sc-649, 1:3,000; Santa Cruz Biotechnology, Inc.) and $\beta$-actin (sc-7210, 1:2,000; Santa Cruz Biotechnology, Inc.) at $4^{\circ} \mathrm{C}$ overnight, followed by incubation with goat anti-rabbit IgG-HRP secondary antibodies (sc-2004, 1:5,000; Santa Cruz Biotechnology, Inc.) for $1 \mathrm{~h}$ at room temperature. The blot images were observed using BeyoECL Moon kit (P0018F; Beyotime Institute of Biotechnology) and analyzed with Image_Lab version 3.0 (Bio-Rad Laboratories, Inc., Hercules, CA, USA).

Statistical analysis. The values are presented as the mean \pm standard error. One-way analysis of variance followed by a Tukey post hoc test was used to analyze the differences between groups. $\mathrm{P}<0.05$ was considered to indicate a statistically significant difference.

\section{Results}

Quercetin protects LVDs and LVDd in a rat model of CPR. CPR rat were treated with $50 \mathrm{mg} / \mathrm{kg}$ quercetin, and LVDs and LVDd levels were measured to evaluate that quercetin protects against CPR. As presented in Fig. 2A, the LVDs of CPR model rats was higher than that of the sham group. However, quercetin significantly inhibited the LVDs of CPR model rats 

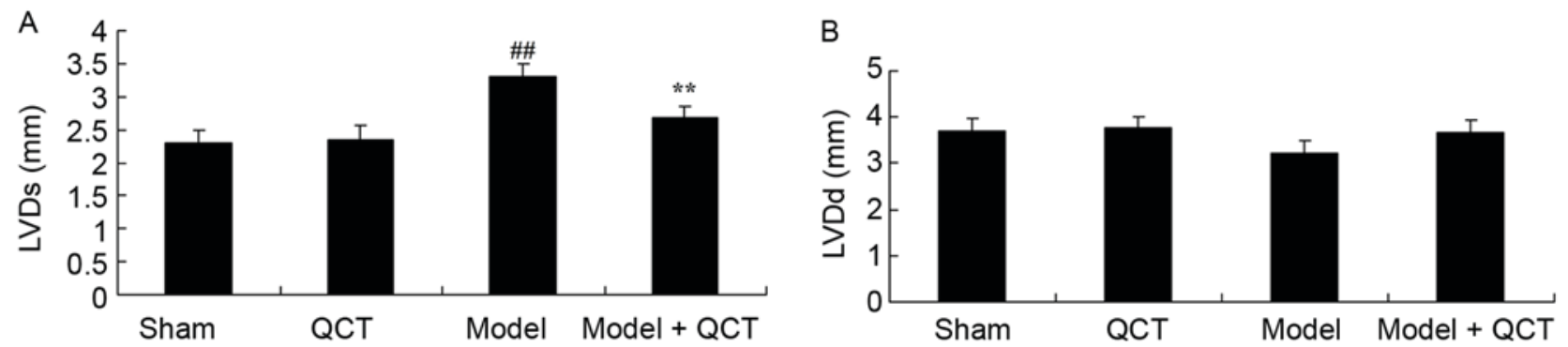

Figure 2. Quercetin protects against LVDs and LVDd levels in rat model of CPR. (A) LVDs and (B) LVDd levels were determined in the rat model of cardiopulmonary resuscitation. ${ }^{\# \#} \mathrm{P}<0.01$ vs. sham group; ${ }^{* *} \mathrm{P}<0.01$ vs. model group. LVDs, left ventricular dysfunction systolic; QCT, quercetin group; Model, CPR model; Model + QCT, CPR model + quercetin group; LVDd, left ventricular dysfunction diastolic; CPR, cardiopulmonary resuscitation .
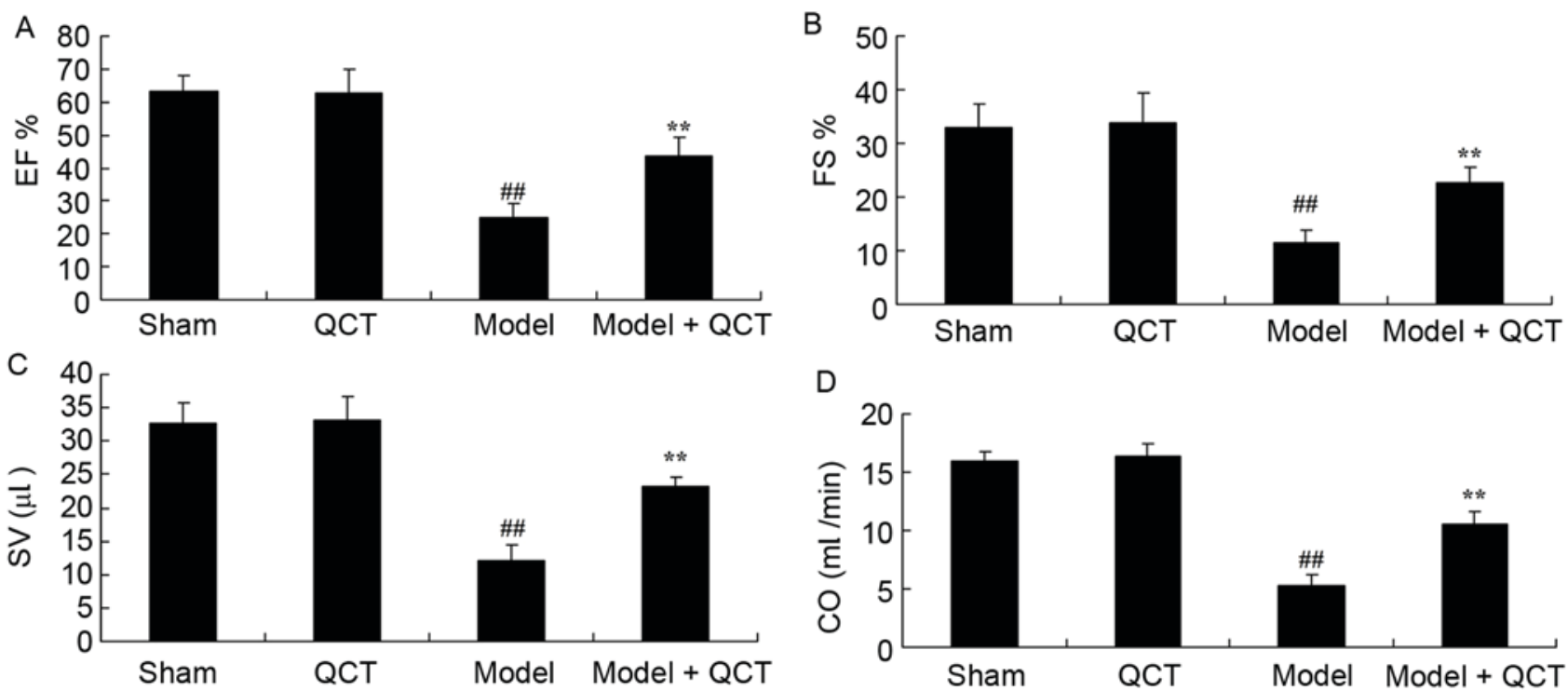

Figure 3. Quercetin protects EF, FS, SV and CO in rat model of CPR. (A) EF, (B) FS, (C) SV and (D) CO in rat model of cardiopulmonary resuscitation. ${ }^{\# \#} \mathrm{P}<0.01$ vs. sham group; ${ }^{* *} \mathrm{P}<0.01$ vs. model group. EF, ejection fraction; QCT, quercetin group; Model, CPR model; Model + QCT, CPR model + quercetin group; FS, left ventricular shortening fraction; SV, stroke volume; CO, cardiac output; CPR, cardiopulmonary resuscitation.

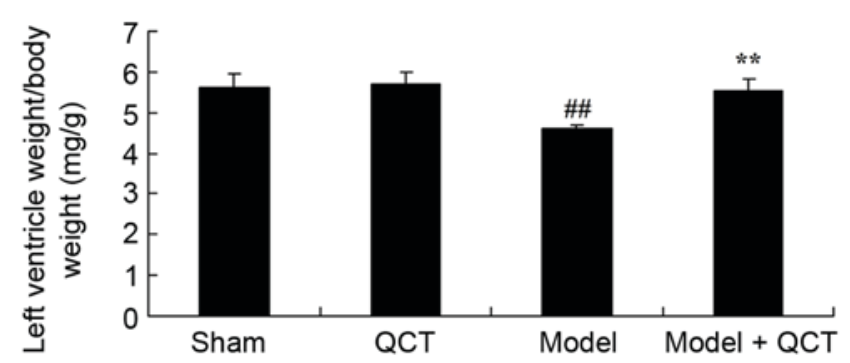

Figure 4. Quercetin protects left ventricle weight/body weight in a rat model of CPR. ${ }^{\# \#} \mathrm{P}<0.01$ vs. sham group; ${ }^{* *} \mathrm{P}<0.01$ vs. model group. QCT, quercetin group; Model, CPR model; Model + QCT, CPR model + quercetin group; $\mathrm{CPR}$, cardiopulmonary resuscitation.

(Fig. 2A). There was no significant difference in the LVDd between the sham, QCT, CPR model group and model + QCT groups ( $\mathrm{P}>0.05$; Fig. 2B).

Quercetin protects the EF, FS, SV and CO in a rat model of $C P R$. It was evaluated whether quercetin protects $\mathrm{EF}, \mathrm{FS}, \mathrm{SV}$

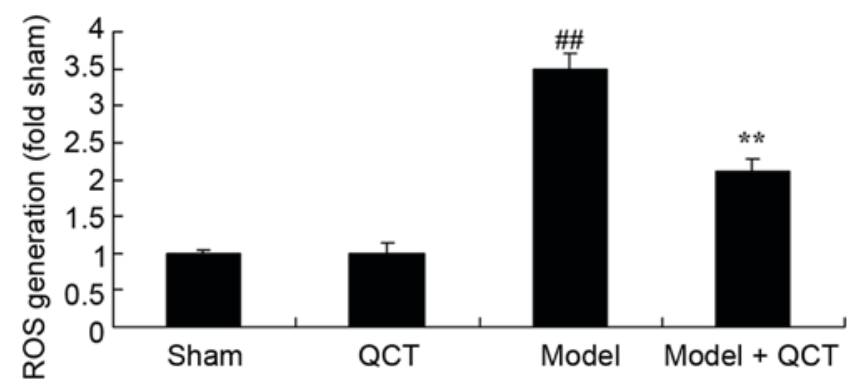

Figure 5. Quercetin protects against ROS generation in rat model of CPR. ${ }^{\# \#} \mathrm{P}<0.01$ vs. sham group; ${ }^{* *} \mathrm{P}<0.01$ vs. model group. ROS, reactive oxygen species; QCT, quercetin group; Model, CPR model; Model + QCT, CPR model + quercetin group; CPR, cardiopulmonary resuscitation .

and $\mathrm{CO}$ in the rat model of CPR. There was no significant difference between the sham group and QCT group (Fig. 3). However, the levels of EF, FS, SV and CO in the CPR model rats were significantly lower than those of the sham group (Fig. 3). Treatment with quercetin significantly increased the EF, FS, SV and CO levels in CPR model rats (Fig. 3). 
A

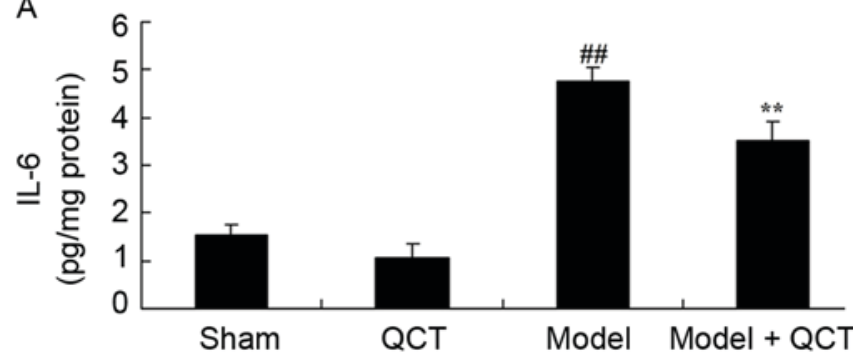

B

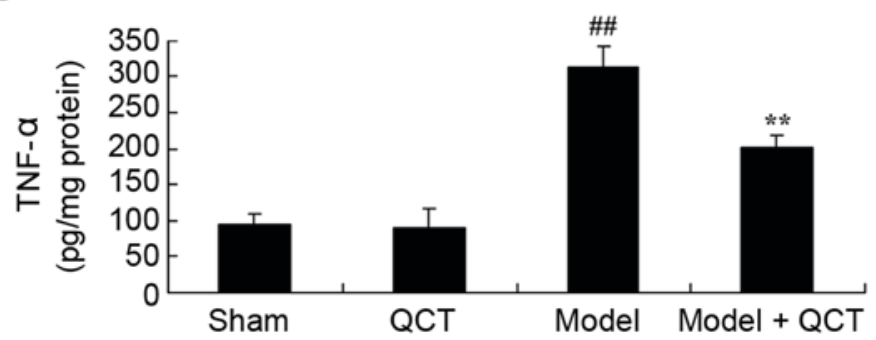

Figure 6. Quercetin protects against inflammation in rat model of CPR. (A) IL-6 and (B) TNF- $\alpha$ were measured in a rat model of CPR. ${ }^{\#} \mathrm{P}<0.01$ vs. sham group; ${ }^{* *} \mathrm{P}<0.01$ vs. model group. IL-6, interleukin; QCT, quercetin group; Model, CPR model; Model + QCT, CPR model + quercetin group; TNF- $\alpha$, tumor necrosis factor- $\alpha$; CPR, cardiopulmonary resuscitation.

Quercetin protects the left ventricle weight/body weight in a rat model of CPR. Subsequently, it was verified whether quercetin protects the left ventricle weight/body weight in a rat model of CPR. As presented in Fig. 4, there was no significant difference in left ventricle weight/body weight between sham group and QCT group. The left ventricle weight/body weight in the CPR model group was significantly lower than that of the sham group (Fig. 4). Treatment with quercetin significantly enhanced left ventricle weight/body weight in CPR model rats (Fig. 4).

Quercetin protects against ROS generation in rat model of cardiopulmonary resuscitation. The results demonstrated that there was no significant change in ROS generation between the sham group and QCT group ( $>>0.05$; Fig. 5). The CPR model significantly induced ROS generation in rats compared with the sham group (Fig. 5). Treatment with quercetin significantly suppressed ROS generation in CPR model rats (Fig. 5).

Quercetin protects against inflammation in rat model of cardiopulmonary resuscitation. To determine the anti-inflammatory effect of quercetin in CPR model rats, IL-6 and TNF- $\alpha$ levels were measured. IL- 6 and TNF- $\alpha$ were similar in the sham group and QCT group (P>0.05; Fig. 6). IL-6 and TNF- $\alpha$ levels were significantly induced in the CPR rat model group compared with the sham group (Fig. 6). Treatment with quercetin significantly inhibited the activation of IL- 6 and TNF- $\alpha$ in CPR model rats (Fig. 6).

Quercetin reduces MMP-2 and iNOS protein expression and induces Bmi-1 protein expression in a rat model of $C P R$. It was evaluated whether quercetin protects against increased MMP-2, iNOS and Bmi-1 protein expression in a rat model of CPR using western blotting analysis. There was no significant difference in MMP-2, iNOS and Bmi-1 protein expression between the sham group and QCT group (Fig. 7). However, CPR significantly induced MMP-2 and iNOS protein expression and suppressed Bmi-1 protein expression in compared with the sham group (Fig. 7). Quercetin treatment significantly inhibited MMP-2 and iNOS protein expression, and induced Bmi-1 protein expression in cardiopulmonary resuscitation rat (Fig. 7).

Quercetin protects against caspase-3 activity in rat model of CPR. The current results revealed that there was no significant difference in caspase-3 activity between the sham group and QCT group ( $\mathrm{P}>0.05$; Fig. 8). Caspase-3 activity was significantly increased in the CPR model group compared with the sham group (Fig. 8). Quercetin treatment significantly inhibited caspase-3 activity in CPR model rats (Fig. 8).

\section{Discussion}

CPR causes hypoxia-ischemia. Effective and timely CPR may restore the heart beat for the majority of patients (20). However, cerebral injury following resuscitation remains difficult issue for complete rehabilitation (21). Following successful CPR, the autonomic circulation recovery of the cerebral blood flow causes cerebral-reperfusion injury and may cause further aggravation of the prognosis of cerebral functions and mortality (22). In present study, quercetin significantly reduced the LVDs, increased the EF, FS, SV and $\mathrm{CO}$, and enhanced left ventricle weight/body weight in CPR model rats.

Following cardiac arrest, organisms are in severe and general hypoxic-ischemic states, which lead to reperfusion injury and secondary lesions of visceral organs (23). During CPR, organisms produce stress reactions. Under strong pathological stimuli, cytokines are produced and cascade reactions are triggered. Reperfusion injury following cardiac arrest is closely associated with pro-inflammatory cytokines, including TNF- $\alpha$, IL-1 and IL-6 (21). The current study demonstrated that treatment with quercetin significantly inhibited the activation of IL- 6 and TNF- $\alpha$ release and suppressed ROS generation in CPR model rats. Liu et al (24) reported that quercetin suppressed insulin-mediated glucose disposal during inflammatory conditions in skeletal muscle tissue/cells.

In cardiac muscle tissues, MMPs and TIMPs are tightly balanced. If the level of MMPs increases, the balance is disrupted, which may cause the remodeling of ECM (25). Previous studies reported that MMPs have an important role in left atrioventricular remodeling following acute myocardial infarction, chronic heart failure, hypertension, diastolic cardiomyopathy and atrial fibrillation $(25,26)$. MMPs also participate in pathophysiological processes, including platelet aggregation, angiotasis regulation, inflammation and ischemia-reperfusion injury (27). However, studies on the 

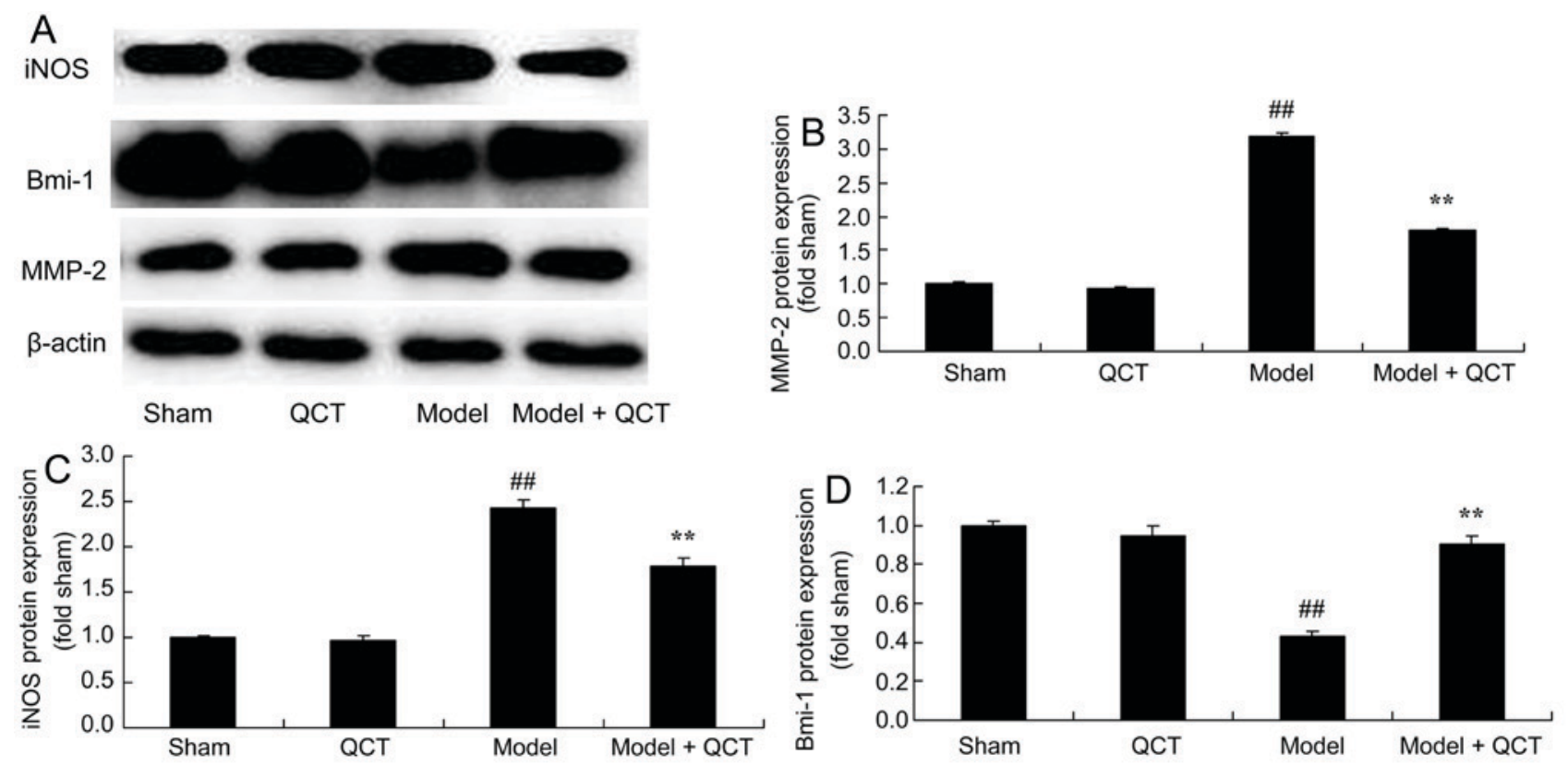

Figure 7. Quercetin reduces MMP-2 and iNOS protein expression and induced Bmi-1 protein expression in a rat model of CPR. (A) Western blotting and (B-D) densitometry analysis of MMP-2, iNOS and Bmi-1 protein expression in a rat model of CPR. ${ }^{\# \#} \mathrm{P}<0.01$ vs. sham group; ${ }^{* *} \mathrm{P}<0.01$ vs. model group. MMP-2, matrix metalloproteinase-2; QCT, quercetin group; Model, CPR model; Model + QCT, CPR model + quercetin group; CPR, cardiopulmonary resuscitation.

association between MMPs and ventricular fibrillation are rare. In the current study, quercetin treatment significantly inhibited MMP-2 protein expression in CPR model rats. Barteková et al (16) demonstrated that quercetin improves post-ischemic recovery of heart function through suppression of MMP-2 and anti-apoptosis.

Reperfusion following CPR can cause server cerebral anoxia and ultimately result in dysneuria. Randomized clinical trial confirmed that low temperature therapy is effective for coma patients following CPR, with ventricular fibrillation to improve dysneuria. It has been reported that fast cooling of the head when conducting CPR can improve survival rates and nervous system functions, while studies on the endogenous protective mechanisms involved are rare (29). Biological behaviors and external information transmission are produced by a series of signal transduction and regulation mechanisms. Signal transduction systems have an essential role in cell differentiation, growth, apoptosis and gene expression (30). In the current study, quercetin treatment significantly inhibited caspase- 3 activity in CPR model rats.

It is generally established that Bmi-1 is highly expressed in hematopoietic stem cells and neural stem cells (31). Bmi-1 is involved in maintaining the self-renewal capacities of stem cells and has an important role in stem cell growth (32). In the current study, treatment with quercetin significantly increased the protein expression of Bmi-1 and suppressed the protein expression of iNOS in CPR model rats. Dong et al (32) demonstrated that quercetin attenuates doxorubicin cardiotoxicity through activation of Bmi-1 expression. Zhang et al (15) suggested that quercetin protected endothelial NOS expression in cavernous endothelial cells.

In summary, quercetin significantly inhibited the LVDs, increased EF, FS, SV and CO, and enhanced left ventricle

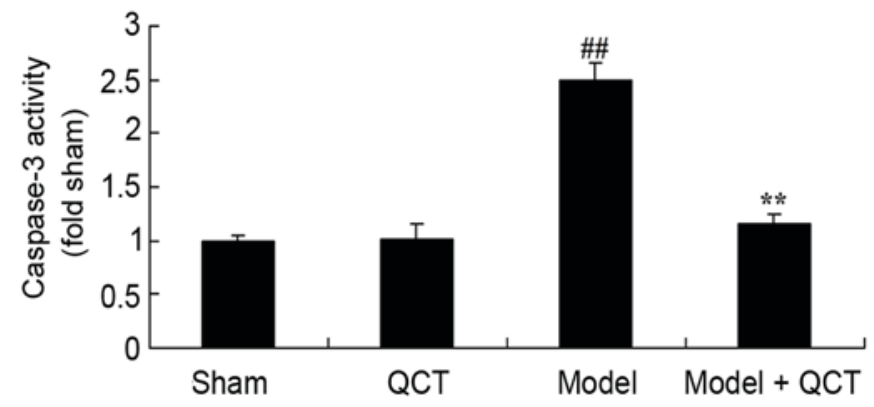

Figure 8. Quercetin reduces caspase-3 activity in rat model of CPR. ${ }^{\# \#} \mathrm{P}<0.01$ vs. sham group; ${ }^{* *} \mathrm{P}<0.01$ vs. model group. QCT, quercetin group; Model, CPR model; Model + QCT, CPR model + quercetin group; CPR, cardiopulmonary resuscitation.

weight/body weight in a rat CPR model. Additionally, quercetin protected against inflammation, MMP-2 activation, iNOS expression and apoptosis, and modulated Bmi-1 expression in rat model of CPR.

\section{Acknowledgements}

Not applicable.

\section{Funding}

Natural Science Foundation of China (grant no. 81471830).

\section{Availability of data and materials}

The analyzed data sets generated during the study are available from the corresponding author on reasonable request. 


\section{Authors' contributions}

X-LL and NZ conceived and designed the experiment; DW, XL, X-MJ and CY performed the experiments; X-LL and NZ wrote the paper. DW, X-LL and NZ analyzed the data.

\section{Ethics approval and consent to participate}

The protocol of animal experiments was approved by the University Laboratory Animal Research Committee of The First Hospital of Jilin University (Changchun, China).

\section{Consent for publication}

Not applicable.

\section{Competing interests}

The authors declare that they have no competing interests.

\section{References}

1. Capuano F, Lechiancole A, Angeloni E, Goracci M, Bianchin R, Roscitano A, Comito C, Melina G and Sinatra R: Miniaturized versus conventional cardiopulmonary bypass in patients undergoing coronary artery bypass surgery: Impact on lymphocyte depletion and sternal wound healing. J Cardiothorac Surg 10 (Suppl 1): A322, 2015.

2. Wang XT, Liu DW, Zhang HM and Chai WZ: Integrated cardiopulmonary sonography: A useful tool for assessment of acute pulmonary edema in the intensive care unit. J Ultrasound Med 33: 1231-1239, 2014.

3. Souza SS, Intelisano TR, De Biaggi CP, Moura CA, Selmi AL, Dias RA and Cortopassi SR: Cardiopulmonary and isoflurane-sparing effects of epidural or intravenous infusion of dexmedetomidine in cats undergoing surgery with epidural lidocaine. Vet Anaesth Analg 37: 106-115, 2010.

4. Wang X, Ji B, Zhang Y, Zhu X, Liu J, Long C and Zheng Z: Comparison of the effects of three cell saver devices on erythrocyte function during cardiopulmonary bypass procedure-a pilot study. Artif Organs 36: 931-935, 2012.

5. Bhutta AT, Schmitz ML, Swearingen C, James LP, Wardbegnoche WL, Lindquist DM, Glasier CM, Tuzcu V, Prodhan P, Dyamenahalli U, et al: Ketamine as a neuroprotective and anti-inflammatory agent in children undergoing surgery on cardiopulmonary bypass: A pilot randomized, double-blind, placebo-controlled trial. Pediatr Crit Care Med 13: 328-337, 2012.

6. Sun YJ, Song DD, Diao YG, Zhou J and Zhang TZ: Penehyclidine hydrochloride preserves the intestinal barrier function in patients undergoing cardiopulmonary bypass. J Thorac Cardiovasc Surg 146: 179-185, 2013.

7. Garner JR, Stroud RE, Finklea L, Ikonomidis JS, Dorman BH and Spinale FG: The effects of leukocyte reduction on matrix metalloproteinase release in cardiopulmonary bypass. J Extra Corpor Technol 36: 185-190, 2004.

8. Smith CR, Stamou SC, Boeve TJ and Hooker RC: Repair of a penetrating ascending aortic ulcer with localized resection and extracellular matrix patch aortoplasty. Ann Thorac Surg 94: 988-989, 2012

9. Asberg AE and Videm V: Neutrophil dysfunction after biomaterial contact in an in vitro model of cardiopulmonary bypass. Eur J Cardiothorac Surg 30: 744-748, 2006.

10. Chowdhury B, Hemming R, Hombach-Klonisch S, Flamion B and Triggs-Raine B: Murine hyaluronidase 2 deficiency results in extracellular hyaluronan accumulation and severe cardiopulmonary dysfunction. J Biol Chem 288: 520-528, 2013.

11. Wang MC, Li CL, Cui J, Jiao M, Wu T, Jing LI and Nan KJ: BMI-1, a promising therapeutic target for human cancer. Oncol Lett 10: 583-588, 2015.

12. Jiang L, Li J and Song L: Bmi-1, stem cells and cancer. Acta Biochim Biophys Sin (Shanghai) 41: 527-534, 2009.
13. Koning NJ, de Lange F, Vonk AB, Ahmed Y, van den Brom CE, Bogaards S, van Meurs M, Jongman RM, Schalkwijk CG, Begieneman MP, et al: Impaired microcirculatory perfusion in a rat model of cardiopulmonary bypass: The role of hemodilution. Am J Physiol Heart Circ Physiol 310: H550-H558, 2016.

14. Link MS, Myerburg RJ and Estes NA III; American Heart Association Electrocardiography and Arrhythmias Committee of Council on Clinical Cardiology, Council on Cardiovascular Disease in Young, Council on Cardiovascular and Stroke Nursing, Council on Functional Genomics and Translational Biology, and American College of Cardiology: Eligibility and disqualification recommendations for competitive athletes with cardiovascular abnormalities: Task force 12: Emergency action plans, resuscitation, cardiopulmonary resuscitation, and automated external defibrillators: A scientific statement from the American heart association and American college of cardiology. Circulation 132: e334-e338, 2015.

15. Zhang Y, Huang C, Liu S, Bai J, Fan X, Guo J, Jia Y, Zhang Z, Chen X, Jia Y, et al: Effects of quercetin on intracavernous pressure and expression of nitrogen synthase isoforms in arterial erectile dysfunction rat model. Int J Clin Exp Med 8: 7599-7605, 2015.

16. Barteková $M$, Šimončíková $P$, Fogarassyová $M$, Ivanová $M$, Okruhlicová L', Tribulová N, Dovinová I and Barančík M: Quercetin improves postischemic recovery of heart function in doxorubicin-treated rats and prevents doxorubicin-induced matrix metalloproteinase-2 activation and apoptosis induction. Int J Mol Sci 16: 8168-8185, 2015.

17. Shen XC, Yang YP, Xiao TT, Peng J and Liu XD: Protective effect of oxymatrine on myocardial fibrosis induced by acute myocardial infarction in rats involved in TGF- $\beta_{1}$-smads signal pathway. J Asian Nat Prod Res 13: 215-224, 2011.

18. Uraoka M, Nakajima Y, Kurita T, Suzuki A, Takata K and Sato S: Landiolol, an ultra short acting betal-blocker, improves pulmonary edema after cardiopulmonary resuscitation with epinephrine in rats. J Anesth 24: 67-72, 2010.

19. Ye S, Weng Y, Sun S, Chen W, Wu X, Li Z, Weil MH and Tang W: Comparison of the durations of mild therapeutic hypothermia on outcome after cardiopulmonary resuscitation in the rat. Circulation 125: 123-129, 2012.

20. Duppen N, Etnel JR, Spaans L, Takken T, van den Berg-Emons RJ, Boersma E, Schokking M, Dulfer K, Utens EM, Helbing W and Hopman MT: Does exercise training improve cardiopulmonary fitness and daily physical activity in children and young adults with corrected tetralogy of Fallot or Fontan circulation? A randomized controlled trial. Am Heart J 170: 606-614, 2015.

21. Durukan AB, Gurbuz HA, Salman N, Unal EU, Ucar HI and Yorgancioglu CE: Ventilation during cardiopulmonary bypass did not attenuate inflammatory response or affect postoperative outcomes. Cardiovasc J Afr 24: 224-230, 2013

22. Guizilini S, Alves DF, Bolzan DW, Cancio AS, Regenga MM, Moreira RS, Trimer R and Gomes WJ: Sub-xyphoid pleural drain as a determinant of functional capacity and clinical results after off-pump coronary artery bypass surgery: A randomized clinical trial. Interact Cardiovasc Thorac Surg 19: 382-387, 2014.

23. Sayed S, Idriss NK, Sayyedf HG, Ashry AA, Rafatt DM, Mohamed AO and Blann AD: Effects of propofol and isoflurane on haemodynamics and the inflammatory response in cardiopulmonary bypass surgery. Br J Biomed Sci 72: 93-101, 2015.

24. Liu K, Mei F, Wang Y, Xiao N, Yang L, Wang Y, Li J, Huang F, Kou J, Liu B and Qi LW: Quercetin oppositely regulates insulin-mediated glucose disposal in skeletal muscle under normal and inflammatory conditions: The dual roles of AMPK activation. Mol Nutr Food Res 60: 551-565, 2016.

25. Wang CT, Zhang L, Wu HW, Wei L, Xu B and Li DM: Doxycycline attenuates acute lung injury following cardiopulmonary bypass: Involvement of matrix metalloproteinases. Int $\mathrm{J}$ Clin Exp Pathol 7: 7460-7468, 2014.

26. He ZJ, Huang ZT, Chen XT and Zou ZJ: Effects of matrix metalloproteinase 9 inhibition on the blood brain barrier and inflammation in rats following cardiopulmonary resuscitation. Chin Med J (Engl) 122: 2346-2351, 2009.

27. Liu K, Shen L, Wang J, Dong G, Wu H, Shao H and Jing H: The preventative role of curcumin on the lung inflammatory response induced by cardiopulmonary bypass in rats. J Surg Res 174: 73-82, 2012. 
28. Minamishima S, Bougaki M, Sips PY, Yu JD, Minamishima YA Elrod JW, Lefer DJ, Bloch KD and Ichinose F: Hydrogen sulfide improves survival after cardiac arrest and cardiopulmonary resuscitation via a nitric oxide synthase 3-dependent mechanism in mice. Circulation 120: 888-896, 2009.

29. Mendoza-Paredes A, Liu H, Schears G, Yu Z, Markowitz SD Schultz S, Pastuszko P, Greeley WJ, Nadkarni V, Kubin J, et al: Resuscitation with $100 \%$, compared with $21 \%$, oxygen following brief, repeated periods of apnea can protect vulnerable neonatal brain regions from apoptotic injury. Resuscitation 76: 261-270, 2008 .
30. ShujaF,Tabbara M,Li Y,Liu B,ButtMU,Velmahos GC,DeMoya M and Alam HB: Profound hypothermia decreases cardiac apoptosis through Akt survival pathway. J Am Coll Surg 209: 89-99, 2009.

31. Wiedemann D, Schachner T, Bonaros N, Weidinger F, Kolbitsch C, Friedrich G, Laufer G and Bonatti J: Does obesity affect operative times and perioperative outcome of patients undergoing totally endoscopic coronary artery bypass surgery? Interact Cardiovasc Thorac Surg 9: 214-217, 2009.

32. Dong Q, Chen L, Lu Q, Sharma S, Li L, Morimoto S and Wang G: Quercetin attenuates doxorubicin cardiotoxicity by modulating Bmi-1 expression. Br J Pharmacol 171: 4440-4454, 2014. 